\title{
Simultaneous Sensing and Actuating for Path Condition Monitoring of a Power Wheel Chair
}

\author{
Hossein Mousavi Hondori \\ Department of Mechanical and \\ Aerospace Engineering \\ University of California, Irvine \\ USA \\ hoss0001@e.ntu.edu.sg
}

\author{
Pham Quoc Trung \\ School of Mechanical and Aerospace \\ Engineering \\ Nanyang Technological University \\ Singapore \\ pham0014@e.ntu.edu.sg
}

\author{
Ling Shih-Fu \\ School of Mechanical and Aerospace \\ Engineering \\ Nanyang Technological University \\ Singapore \\ msfling@ntu.edu.sg
}

\begin{abstract}
Monitoring the ambience's condition is emerging as one of the most important issues in mobile robots. While we need to detect and process the condition quickly and efficiently using sensors, they (sensors) introduce extra complexity to the systems. Therefore this paper aims to develop a new method to simplify the sensing process. It describes an experimental process for monitoring path conditions of a power wheel chair inside and outside of the lab. The monitoring method triggers impedance via transduction matrix of the DC motors which run the wheelchair. The matrix is based on the relationship between electrical and mechanical impedance of the motors. A voltage probe and a current probe are used to monitor the changes of voltage and current supplied to the two DC motors of the system while the wheel chair is moving and the signals are recorded by a Digital Signal Analyzer (DSA). The recorded data are processed by MATLAB and the graphs representing various conditions are plotted. The relationship between the conditions of the path and the changes of voltage, current, and impedance of the motors are listed. The use of the method is justified but miniaturization of the voltage and current probes is necessary for real-life implementation of the system.
\end{abstract}

\section{INTRODUCTION}

Impedance control is a concept proposed by Neville Hogan [1] which concerns robotics. It regards the importance of force control and in some way disregards the force trajectory. In other words it emphasizes on how the mechanical impedance is controlled and regulated. To implement impedance control, it is essential

to use both velocity and force sensors. This adds up to the cost and intricacy of the systems. Anderson et al [2] used an approach to measure the mechanical impedance of a PZT coated cantilever beam derived from the electro-mechanical modeling of the actuator. The simultaneous sensing and actuating (SSA) was able to successfully measure and control the vibration of the PZT-coated beam. Ling et al [3], [4] were able to further advance the method by removing the loading effect caused by the shaker without solving the governing equations. Further more in $[5$ 